\title{
Deep Reinforcement Learning in Human Activity Recognition: A Survey
}

This paper was downloaded from TechRxiv (https://www.techrxiv.org).

\section{LICENSE}

CC BY 4.0

SUBMISSION DATE / POSTED DATE

$14-02-2022$ / 22-02-2022

CITATION

Nikpour, Bahareh (2022): Deep Reinforcement Learning in Human Activity Recognition: A Survey. TechRxiv. Preprint. https://doi.org/10.36227/techrxiv.19172369.v1

DOI

10.36227/techrxiv.19172369.v1 


\title{
Deep Reinforcement Learning in Human Activity Recognition: A Survey
}

\author{
Bahareh Nikpour ${ }^{1,2}$, Dimitrios Sinodinos ${ }^{1,2}$, Narges Armanfard ${ }^{1,2}$ \\ ${ }^{1}$ McGill University, Montreal, Quebec, Canada \\ ${ }^{2}$ Mila-Quebec AI Institute, Montreal, Quebec, Canada \\ bahareh.nikpour@mail.mcgill.ca, dimitrios.sinodinos@mail.mcgill.ca, narges.armanfard@mcgill.ca
}

\begin{abstract}
Human activity recognition is a popular research field in computer vision that has already been widely studied. However, it is still an active research field since it plays an important role in many current and emerging real world intelligent systems, like visual surveillance and humancomputer interaction. Deep Reinforcement Learning (DRL) has recently been employed to address the activity recognition problem with various purposes, such as finding attention in video data or obtaining the best network structure. DRL-based human activity recognition has only been around for a short time, and it is a challenging, novel field of study. Therefore, to facilitate further research in this field, we have constructed a comprehensive survey on activity recognition methods that incorporate deep reinforcement learning. Towards the end of this survey, we summarize key challenges and open problems in this area that can be addressed by researchers in the future. ${ }^{1}$
\end{abstract}

\section{Introduction}

Human activity recognition (HAR) is a challenging research field dealing with identifying and recognizing the activities or movements performed by humans. While humans can easily recognize activities they observe in videos, fully automating this procedure is quite difficult, yet crucial due to its extensive real-world applications, such as video surveillance, human-robot interaction, fitness monitoring, sports analysis, and activity monitoring in elderly people. Appropriate data can be captured by different available sensors ranging from RGB and depth cameras [Wang et al., 2015; Tang et al., 2018a] to wearable sensors such as accelerometers and gyroscopes [Wang et al., 2016]. Hence, various modalities can be used for HAR, such as: RGB data that is the most commonly used modality, skeleton data that contains 2 or 3 dimensions of body joint locations, radar, and WiFi [Sun et al., 2020].

In the computer vision literature, human activity recognition is formulated as a learning problem, and machine

\footnotetext{
${ }^{1}$ This work has been submitted for possible publication. Copyright may be transferred without notice, after which this version may no longer be accessible
}

learning tools are employed to classify and recognize the activities. Before the emergence of Deep Learning (DL) models in activity recognition techniques, researchers were mainly focused on developing hand-crafted features, such as interest points and motion descriptors, that are capable of capturing the information in videos [Laptev et al., 2008; Klaser et al., 2008]. Since extracting hand crafted features is labor intensive and requires domain knowledge, DL methods started being introduced to the field for extracting effective video representations and hence increasing generalization. This resulted in a significant progress in the field.

Reinforcement learning (RL) is a sub-field of machine learning that, unlike supervised learning methods, learns in an unsupervised fashion through the interaction of one or multiple agents with an environment [Sutton and Barto, 2018]. These interactions result in either positive or negative rewards based on a defined reward function. By trial and error over time, more information is acquired from the environment and the agent learns how to maximize the expected reward, without knowing the ground-truth. More recently, reinforcement learning has been incorporated in deep learning; creating a new class of algorithms called Deep Reinforcement Learning (DRL), which its effectiveness is demonstrated in many applications [Liu et al., 2019; Shao et al., 2019]. DRL enables RL agents to make decisions using the unstructured input data without manually engineering the state space. It is mainly used to deal with high dimensional state and action spaces, which are prevalent in most of the practical real world problems such as video game playing [Torrado et al., 2018] and self-driving cars control [Sallab et al., 2017].

The progress and success of deep reinforcement learning methods has encouraged researchers to use it in computer vision applications in order to address the problems of large state and action spaces, which cannot be addressed with traditional RL methods. There are many DRL-based studies in the field of computer vision such as video captioning [Wang et al., 2018], visual tracking [Yun et al., 2017], activity detection [Yeung et al., 2016], activity localization [Wang et al., 2019], face recognition [Rao et al., 2017], and person identification [Haque et al., 2016]. However, there are a few studies on DRL-based activity recognition. In the recent DRL-based activity recognition works, DRL is employed for various purposes such as attention finding and optimizing the network's structure, to improve the recognition 
performance. It is mainly used in scenarios where general deep learning methods cannot be used, e.g. when groundtruth is not available, or if the designed objective function is non-differentiable. There are several survey papers for activity recognition [Herath et al., 2017; Sarkar et al., 2022; Kong and $\mathrm{Fu}, 2018$ ], but none of them have covered reinforcement learning in the HAR task. Although there are a few RL-based HAR researches, it is worth having an overview on them to evaluate the effectiveness of RL in the field, to investigate their pros and cons, and to provide insight for future researchers in the field. The focus of this study is on providing a comprehensive review of various DRL-based activity recognition methods and discussing the challenges for future works.

Note that to the best of our knowledge, there are only two studies [Yamagata et al., 2019; Ji et al., 2018] that use traditional RL methods, i.e. non-deeplearning based RL, for HAR as a small number of RL states/actions are defined in theses studies. However, such regular RL algorithms are not effective when there a large number of states/actions like in all other DRL-based HAR methods.

The paper is organized as follows. We briefly discuss deep reinforcement learning in Section 2. In Section 3 we dive into specific DRL-based activity recognition methods. Next, we will present some challenges and open problems in Section 4 , before finally offering some insightful conclusions.

\section{Deep Reinforcement Learning}

Inspired by the way humans learn to behave optimally in different environments, reinforcement learning algorithms learn to perform a specific task and attain a complex objective through interaction with an environment [Sutton and Barto, 2018]. In every RL algorithm, there exist an agent that explores the environment to learn a policy, and receives a reward signal aligned with the agent's final goal(s). The agent's intention is maximizing an expected reward, which is known as the Value. RL problems are usually modeled as a Markov Decision Process (MDP), that can be expressed by a triple $(S, A, R)$, where $S$ denotes the state of the environment, $A$ is the set of all possible actions ${ }^{2}$ and $R$ represents the reward. In one step of an RL episode, the agent performs an action, receives a reward and moves to a new state. The reward is then used to update the policy so that at the end, the agent finds its optimal behavior (i.e., the policy).

Deep reinforcement learning is a group of $\mathrm{RL}$ techniques that use deep neural networks, which are powerful function approximators, to deal with high dimensional action/state spaces [Li, 2017; Arulkumaran et al., 2017; François-Lavet et al., 2018]. Deep RL has proven to be very successful, as demonstrated by [Mnih et al., 2013; Mnih et al., 2015; Jaderberg et al., 2019; Nguyen et al., 2017]. There are two main categories of DRL techniques: methods based on value-function and methods based on policy gradient. Methods of the first category try to estimate the value of each state and determine the optimum policy by finding the maximum state value function. The optimum policy, shown by

\footnotetext{
${ }^{2}$ As there are two types of actions in this survey, i.e. the human action and the RL action, we refer to the human action as activity everywhere, for clarity.
}

$\pi^{*}$, corresponds to the optimal state value function $V^{*}(s)$, where $s$ is the the environment's current state. Deep QNetwork (DQN) is one of the most popular methods in this category, which involves incorporating deep learning into the traditional Q-learning. DQN has achieved superhuman results in some Atari games [Mnih et al., 2013]. On the other hand, policy gradient methods parameterize the policy and try to directly optimize it with respect to the expected reward. Monte carlo policy gradient (REINFORCE) and Actor-Critic are the two popular methods of this category [Sutton and Barto, 2018]. The difference of Actor-Critic with REINFORCE is that instead of waiting until the end of an episode to get the cumulative reward, it uses bootstrapping.

In general, there are many trade-offs to consider when comparing the value-based and policy gradient methods [Sutton et al., 2000]. For instance, policy gradient methods can be applied to a broader range of problems - e.g. when considering how deep Q-learning fails when Q-functions are too complex. Also, policy gradient methods usually converge faster, but they tend to converge to local optimum. DQN cannot learn stochastic policies, but policy gradient methods can model probabilities of actions. Last but not least, policy gradient methods are capable of modeling a continuous action space, while DQN uses an expensive action discretization process.

\section{Deep Reinforcement Learning in Activity Recognition}

In this section, DRL-based human activity recognition methods are reviewed. In all approaches, there is a task that can be considered as a search problem with no ground-truth of the optimal solution. Consequently, the solution should be detected by interacting with the environment, and the problem can be perfectly modeled by reinforcement learning and solved by DRL techniques. The summary of methods is presented in Table 1.

\subsection{Temporal attention finding}

As a parallel to RL being inspired by human learning, attention mechanisms in intelligent systems share the benefits of human-drawn attention, where focusing on key elements of the learning stimulus leads to improved learning. There are two types of attention in this area: soft attention and hard attention. In the soft attention mechanism, some weights from a continuous range of values are assigned to different parts of data based on their importance in making the final decision. On the other hand, hard attention mechanism aims at keeping the most informative input portions and discarding the irrelevant ones; meaning assigning either a weight of 0 or 1 to the portions. For video sequences, temporal attention refers to defining the most informative frames that can significantly improve the recognition accuracy compared to the case of using all frames for recognition. There are several works in activity recognition where deep reinforcement learning is used to find the temporal attention. RL is beneficial in this context as there are numerous frames in each video and there is no specific ground-truth on which frames are informative. In this scenario, an RL agent explores and interacts with the environment to find the optimal set of frames for the purpose of HAR. 
Tang et al. proposed a deep progressive RL framework, called DPRL, where an agent progressively seeks the most informative frames in a skeleton video [Tang et al., 2018a]. A fixed number of frames are chosen based on two criterion: the discriminative ability of the selected frames, and the relationship between the selected frames and the entire activity sequence. To consider the human body's topology, the skeleton at each frame is modeled as a graph and a graphbased convolutional neural network is employed to learn the representation. The RL state includes the representation of the whole video, the video with the current selected frames, and a binary mask indicating which frames are selected. The state is given to an agent consisting of a fully connected layer followed by a Softmax function to define the actions. There are three possible actions for each frame selection: move to the right (i.e. select the next frame on the right), move to the left (i.e. select the next frame on the left), and stay (i.e. keep the currently selected frame). After taking the action, a reward determined by a trained baseline classifier is given to the agent. A large positive reward is given if the output class label changes from incorrect to correct. A large negative reward (punishment) is fed to the agent if the change is from correct to incorrect. If the class label does not change, $a+1(-1)$ reward is given to the agent depending if the probability of the correct class is increased (decreased). A policy gradient algorithm is used to train the RL agent.

Frame selection is also helpful when the video data is untrimmed; meaning there are several subsequent activities in a single video or there exist many frames in the video which are not relevant to the ground-truth activities. Such data poses many challenges on video recognition models. Additionally, frame selection is crucial in untrimmed video analysis since feeding all the frames to the the learning model makes the computations very heavy. In [Wu et al., 2019], Wu et al. modeled the frame sampling as multiple MDPs, where each of them is associated with an agent responsible for selecting one frame. The problem is solved by multi-agent reinforcement learning. The environment, i.e. the video, is encoded by a Convolutional Neural Network (CNN) followed by a recurrent neural network to capture the context information. Then, the encoded data is given to a policy network, which includes a fully connected layer followed by a Softmax, to generate actions. The action set of each agent is moving back, moving forward, and no change. When the action for all the agents is no change, it means it is the end of the episode. The reward is generated based on the output of a trained baseline classifier. More specifically, the reward is calculated based on the change of the classifier's confidence toward predicting the correct class. The REINFORCE algorithm is used for the optimization process.

Rather than treating all frames in a video equally, [Dong et al., 2019] proposed using attention-aware sampling to preserve only the frames where discriminative activities occur and discard all the irrelevant frames. This approach can be applied to all existing DL-based activity recognition models. Due to the non-differentiability of the objective in the hard attention finding, the model cannot be trained in an end to end manner. Consequently, the authors defined the frame selection problem as a MDP and used deep reinforcement learning to train the attention model. The RL agent is a bidi- rectional LSTM (BiLSTM) that takes a state at each time step as input. The state consists of features extracted from the given frame and the class label prediction calculated by a pre-trained baseline classifier. The BiLSTM is topped by a fully connected network which its output passes through a Sigmoid function in order to generate action selection probabilities. The frame selection action is the result of sampling a Bernoulli distribution using the aforementioned probabilities to either select or discard a given frame. To find the optimized policy, REINFORCE is used as the RL method and the reward is taken from the pre-trained baseline classifier.

For skeleton-based activity recognition, [Xu et al., 2020] proposed a feature selection network (FSN) with ActorCritic RL algorithm. This is to select the most descriptive frames and discard ambiguous frames in a sequence. The features extracted for each frame of the skeleton sequence are generated by a generalized graph convolutional network (GGCN). The authors showed that their proposed GGCN achieves better feature extraction compared to the other existing graph convolutional networks mainly because GCNN is able to capture latent dependencies among the body joints. In other words, GGCN captures both local and global joint movements. At the end, features of the selected frames are fed into a classifier to generate the final recognition. The FSN contains a policy network and a value network which are actor and critic, respectively. Both networks are based on LSTM. The policy network receives the MDP's state as the input and outputs a distribution of actions. The state at each video time step consists of the features extracted from the current frame and the global features of the whole video. The sampled actions are keeping and removing each frame, and the RL reward is obtained from the classifier; it is 0 if the classification is wrong and if the classification is correct, the reward will be equal to the confidence of the classier. The value network (critic) estimates the expected reward which is essentially the value of each state. Structurally, the value network is built similarly to the policy network (i.e. several fully connected layers and an LSTM), but only produces a single output value.

\subsection{Spatial attention finding}

In the HAR task, finding spatial attention refers to the concept of finding the most informative regions of frames, which results in providing a more accurate recognition.

In different human activities, different body parts are involved. As such, in the skeleton data, the body joints are not equally important in all activities. Motivated by this, a deep reinforcement learning-based method is introduced in [Nikpour and Armanfard, 2021], which selects the most informative joints in skeleton video frames through a spatial hard attention finding approach. For an activity, the selected joints may vary across frames. The process of finding the most important joints is formulated as an MDP and is solved via REINFORCE algorithm. In this approach, the agent, which is a bidirectional LSTM, takes the joints' locations and motions as the input state, and generates a probability vector for each frame that is used to specify actions of DRL. The action is sampled from a Bernoulli distribution of the probabilities, which is either 0 or 1 . A J-dim indicator vector is assigned to each frame as the vector of actions, where $\mathrm{J}$ is 
the number of body joints. Action 1 means that if the corresponding joint was removed in the previous RL step, return it to the selected joint set and if it is already among the selected joints, remove it. Action 0 means no change should be applied. The reward is given by a pre-trained classifier, which is similar to the reward function used in [Tang et al., 2018b].

Most of the activity recognition methods are designed for the videos captured from a third-person by static cameras. There are few works on the videos of first-person view recorded by wearable cameras. Such data is called egocentric. As is mentioned in [Lu et al., 2019], there are high resolution video data in many cases which demand lots of computational resources to be processed. To address this problem in the domain of activity recognition by egocentric video, a DRL-based approach is presented in [Lu et al., 2019], which aims at finding the region of interest (ROI) in each frame of the video. The problem of finding ROI can also be considered as a spatial attention finding problem. To find the ROI, a fixed size bounding box is considered and the problem of finding the best location is modeled as a RL problem. The current frame of the video is given to an agent as its input state and the action includes two real values defining the shifting amount that should be applied to the bounding box location, horizontally and vertically. The Actor-Critic method is used to maximize the accumulated reward received by the classifier. After defining the best region of interest, a deep network is employed to process it while the whole frame is processed by a shallow network. The results showed that they could significantly decrease the computational complexity while retaining the accuracy.

[Wang et al., 2017] is another method that uses DRL as a tool for generating visual attention maps in video frames as a means of obtaining better activity recognition rates. They aim to develop an attention procedure through RL that closely resembles human's jumping and glimpse mechanism. Specifically, at each video frame, a feature cube is generated by a CNN as input to a REINFORCE attention agent. This agent performs a fixed number of jumps over the cube slices to find a weight distribution, which determines the region of interest in each slice. The found attention maps in the slices are then summed up to serve as the input of an LSTM network for the final activity recognition. The same LSTM is used to generate the reward which is formed by considering both the number of true positives and false positives in order to learn high precision.

\subsection{Early recognition}

In some applications like video surveillance, it is not practicable to wait till the end of activity to perform the recognition task. Therefore, there are some methods that aim to recognize an activity based on the videos of unfinished activities. This is called early activity recognition. A lot of activities are similar to each other at their beginning stages, so early activity recognition is very challenging and most of the general activity recognition methods perform weakly in such cases. In [Xiaokai et al., 2019], deep reinforcement learning is used for early activity recognition. The authors introduced a predictability score which defines how accurate the input sequence of an unfinished activity can be rec- ognized. Their method has two steps. First, they train an evaluator for predictability estimation, where an RL agent is employed due to inadequacy of supervision. Second, a classifier is adaptively optimized based on the predictability of different sequences. In the first step, a CNN is used to extract features out of the frames. Then an LSTM followed by a fully connected network is used as the RL agent which receives the extracted features as the state and outputs a probability defining the predictability score for each video frame; therefore, this method can also be considered as temporal attention finding. Afterwards, a Bernoulli distribution with the obtained probabilities is sampled to obtain actions with values 0 or 1 . The action shows whether the frame is beneficial for the prediction ability of its previous frame sequence (one) or not (zero). The reward is designed such that as few predictable frames as possible are selected at the end. Therefore, the reward consists of two parts: one is based on the classifier's accuracy and the other one is based on the cardinality of the selected frame set. REINFORCE algorithm is employed to train the agent. The authors concluded that the predictability scores are highly advantageous to the early recognition task.

In another work in the DRL-based early activity recognition field, inspired by the human recognition behavior, the authors hypothesized that excluding irrelevant classes during the recognition can positively affect the performance [Weng et al., 2020]. This is due to the fact that the difference between the positive class and the negative classes is not the same for all negative classes. Therefore, the negative classes should not be treated similarly. In this paper, first, a classifier is pre-trained on all the classes and then a masking operation is applied on the classifier's probability outputs. The mask determines which classes of data should be kept and which ones should be removed. This process of finding the best combination of negative classes that should be excluded from the training set is modeled as a reinforcement learning problem since there is no ground-truth for the optimal set of excluding classes. In this Markov Decision Process, the state is defined as the concatenation of the features extracted from the observed video frames up to the current time step, the exclusion mask determined by the agent in the previous time step, and the current probability distribution output of the baseline classifier. The action is generated by a neural network agent that is either 0 and 1 for each class, where 1 denotes the corresponding class should remain and 0 means the corresponding class should be excluded. The reward is designed in a way that the negative classes with high classification probability output get excluded and the rest of negative classes remain. Hence, a positive reward equivalent to the probability output of the classifier is given for the negative classes and a punishment is enforced when the class is positive. REINFORCE algorithm is employed for optimization.

\subsection{Other tasks}

WiFi signal is another data modality for activity recognition. The phase and amplitude of Channel State Information (CSI) measured by WiFi receivers and transmitters change if motions occur around them. Such variations can be used as the input of a machine learning based method to detect and rec- 
ognize human activities. However, it is important that the used method should be independent of the WiFi device's location and the subject's orientation and location. Ma et al. proposed a method which does not depend on the location and person, where a $2 \mathrm{D} \mathrm{CNN}$ is used as the recognition model. A reinforcement learning agent is employed to find the best architecture for the recognition model [Ma et al., 2021]. NASCell [Zoph and Le, 2016] is used as the RL agent, which employs a LSTM-based network for neural architecture search. The RL action is the architecture of the recognition model, i.e. CNN. The current architecture of the recognition model is fed to the agent as the current state, and the training accuracy is considered as the reward. Monte Carlo policy gradient method is used to optimize the agent. The authors demonstrated that the recognition performance is significantly improved as the result of using RL for architecture optimization. It is worth noting that RL is widely used as a tool to find network structures in many applications [Zoph and Le, 2016; Jaafra et al., 2019; Gao et al., 2019].

There has been some activity recognition research that use multiple modalities of data as a means to include more information to the model, leading to improvements in the activity recognition performance [Imran and Raman, 2020; Al-Amin et al., 2019]. Such methods mainly deal with multiplexing of the same network model or combining different ones to reach a single decision from different modalities. Each data modality has distinct advantages in recognizing different activities. Gua et al. introduced a DRL-based framework to fuse multimodal data for the purpose of activity recognition [Guo et al., 2021]. The data modalities are RGB, skeleton, and depth videos. In addition, they consider optical flow extracted from the RGB and depth videos as extra modalities. As there is no ground-truth for the optimal set of fusion weights, deep reinforcement learning is employed to dynamically find the best weights. Each modality has its individual network that assigns an effectiveness score to the modality. Then the scores are weighted by an RL agent, and fused to reach the final decision. In the RL framework, the state includes the set of recognition scores of the different modalities, and the weights assigned to them in the previous episode. The RL action is the adjustment needed to be applied on the current fusion weights and the RL reward is designed based on the classification performance after fusing weighted results of the multimodal data. Twin Delayed Deep Deterministic Policy Gradients (TD3) is employed to optimize the RL problem, where a feedforward neural network is used as the structure of both actor and critic.

Activity recognition methods typically require lots of training data for each activity class to work properly. However, it is not practical to gather hundreds or thousands of labelled data to train the network for a new class that had not been seen by the network during the original training phase. This problem has tried to be addressed in zero-shot learning framework where training is performed using the set of seen data classes and testing is applied on some unseen ones. Gowda et al. introduced a method called CLASTER where they take advantage of both the visual embedding of the video data and semantic embedding of their corresponding labels, and use clustering to learn meaningful represen- tation for the videos [Gowda et al., 2021]. The cerntroids of cluster embeddings are used as data representation which map the seen training classes to new unseen classes. The centroids are preferred as they are more robust over outliers. In CLASTER, finding the optimized cluster centroids is modeled as a reinforcement learning problem. They used REINFORCE and designed it's loss in such a way that the agent aims to minimize the Euclidean distance between the cluster centroids and the input data. A reward 1 is given if the sample is classified correctly; otherwise, it is -1 . The results of this method showed that using DRL in centroids finding can greatly increase the purity of data clusters and the capability of the clustering method in differentiating the similar classes.

In the near future, it is predicted that robots will become prevalent in homes. So it would be beneficial to mount sensors on them to be capable of recognizing activities of the home residents. In this case, the location of the robot is important in capturing informative data. To control the robot's position, in [Kumrai et al., 2020], a deep Q-network is employed. The network optimizes the robot's location to maximize the recognition performance. The camera mounted on the robot captures images that are then used to extract skeleton data. The skeleton data and the portions of the images that correspond to the locations of the user's hands are used as the RL state. The RL action set of the robot includes: move to the right, move to the left, and stay. The reward is obtained based on the activity recognition performance, energy consumed by the robot, and robot's distance from the person.

The DRL-tasks for human activity recognition discussed above are visualized in figure 1 .

\section{Conclusion and Research Directions}

In the previous section, we provided a review of various activity recognition approaches that employ deep reinforcement learning to improve the human recognition performance. Most of these methods aim at finding attention, specially temporal attention in videos. Finding attention is a very helpful strategy to boost the classification results by focusing on the most important information. Using deep RL for finding attention has several benefits. Firstly, it does not need any extra label to supervise where the informative regions are located. Instead, the RL agent tries to locate those regions by interacting with the video and a baseline classifier. It can be considered as a weakly supervised method where the reward acts as the supervisor. Secondly, if the goal is finding hard attention, the neural network model becomes non-differentiable and therefore cannot be trained in an end-to-end manner. In this case, using DRL methods is advantageous. Thirdly, unlike most of the supervised attention finding methods, a DRL-based attention finding framework can be used with any baseline recognition model and is compatible with most of data modalities.

As can be seen in Table 1, other than finding attention, DRL has been used for other tasks, such as finding the best network structure and defining the optimal set of weights for data modalities fusion. All these tasks can be considered as a search problem in a large space without the presence of ground-truth. However, a reward signal can guide 
Table 1: Summary of the DRL-based HAR methods, including the DRL task that indicates the purpose of searching using DRL, the modality of the input data, the employed optimization technique, and the publication year.

\begin{tabular}{|c|c|c|c|c|}
\hline DRL-based method & DRL task & Data modality & Algorithm & year \\
\hline [Wang et al., 2017] & ROI & RGB video & REINFORCE & 2017 \\
\hline [Tang et al., 2018a] & Frame selection & Skeleton video & REINFORCE & 2018 \\
\hline [Wu et al., 2019] & Frame selection & RGB untrimmed video & REINFORCE & 2019 \\
\hline [Dong et al., 2019] & Frame selection & RGB video & REINFORCE & 2019 \\
\hline [Xiaokai et al., 2019] & Sequence predictability & RGB video & REINFORCE & 2019 \\
\hline [Lu et al., 2019] & ROI & Egocentric RGB video & Actor-Critic & 2019 \\
\hline [Xu et al., 2020] & Frame selection & Skeleton video & Actor-Critic & 2020 \\
\hline [Weng et al., 2020] & Class exclusion & RGB video & REINFORCE & 2020 \\
\hline [Kumrai et al., 2020] & Robot control & RGB image & REINFORCE & 2020 \\
\hline [Nikpour and Armanfard, 2021] & Joint selection & Skeleton video & REINFORCE & 2021 \\
\hline$[$ Gowda et al., 2021] & Cluster centroids & RGB video & REINFORCE & 2021 \\
\hline [Guo et al., 2021] & Fusion weights & RGB, skeleton, depth videos & TD3 & 2021 \\
\hline [Ma et al., 2021] & Network architecture & WiFi signal & REINFORCE & 2021 \\
\hline
\end{tabular}

the search; making DRL a viable solution. Due to the superiority of the policy gradient methods, such as converging faster or being able to model continuous action spaces, policy gradient methods have been mainly used in the DRL-based HAR methods. Although these methods tend to converge to a local optimal solution, they can still accomplish the desired task and improve the recognition performance. Another commonality among the reviewed methods is that the reward is designed based on a feedback from a pre-trained baseline recognition model.

Using deep reinforcement learning in human activity recognition is a novel field of research that first appeared in 2017. There are still lots of challenges and open problems to be addressed in the future. In the following, some of these challenges are discussed.

Unsupervised activity recognition: Even while being more practical due to the lack of labeled data in real-world problems, there are much fewer unsupervised activity recognition methods compared to the supervised ones that require a huge number of labeled data. In addition to their applicability to the real-world problems, unsupervised methods do not need re-training when new activities are introduced to the training set. In clustering, finding an effective representation plays an important role, therefore, attention finding can significantly help the method to find a more meaningful representation for the data. As mentioned, DRL is a suitable tool when attention finding is considered, so DRL has a great potential of being employed in the unsupervised HAR context in order to improve the performance. The key difference between designing DRL-based supervised and unsupervised learning methods is the RL reward. In DRL-based supervised activity recognition research, the reward always comes from a trained baseline classifier, which is not available in the unsupervised learning. Therefore, an interesting topic for further research would be addressing the challenge of designing the reward function for unsupervised activity recognition.
Computational cost: Integrating DRL into activity recognition makes the training phase more computationally expensive, since the agent's deep network(s) imposes extra trainable parameters to the system, on top of those of the baseline classifier used for the RL reward generation. As long as the training can be done online and the required computational resources are available, the computational complexity would not be problematic. However, many realworld applications require online training, with untrimmed videos. Moreover, with the recent advancements in portable hardware technology and the Internet-of-Things (IoT), wearable devices with sensors are becoming feasible tools for automatic activity recognition. Traditionally, IoT devices, such as microcrontrollers, transmit data to remote systems that have significantly greater computational capabilities. As edge devices are becoming more powerful, they become more capable of performing significant computations locally. This eliminates the need for the high cost data transmission to the cloud and leads to net positive energy savings. To be able to use the DRL-based activity recognition methods for such applications, it is important to optimize the structure of the DRL methods to run on computationally constrained devices.

Problem formulation: The most critical element in a reinforcement learning framework is related to how the RL problem is defined. This involves defining the state, the actions, and more importantly, the reward supervising the agent. All of the existing DRL-based activity recognition methods have simple definitions of state, actions and rewards. It is beneficial to investigate and design more innovative states, actions, and rewards. For example, some intermediate goals can be introduced that will modify the reward and increase the exploration. Moreover, more classification output details may be considered in defining the reward. For example, when the confidence of a classifier towards predicting the correct class increases, a reward should be given to the DRL agent. However, it is still important whether the 


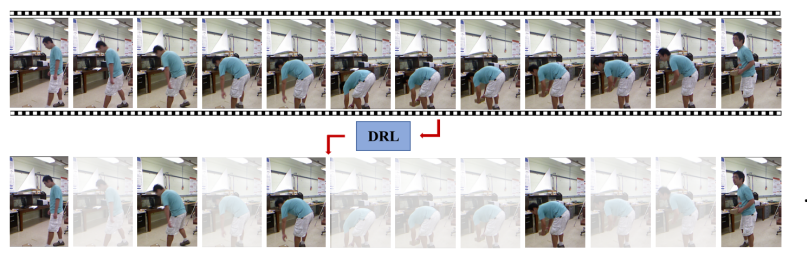

(a)

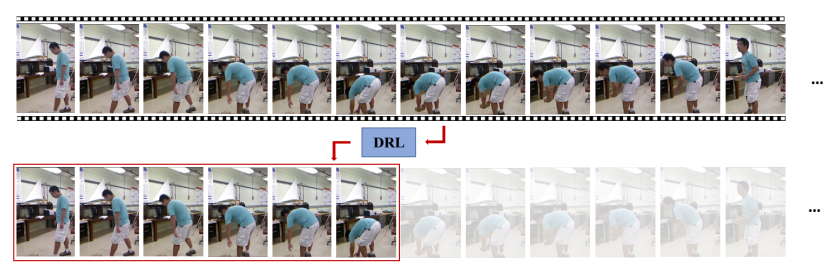

(c)

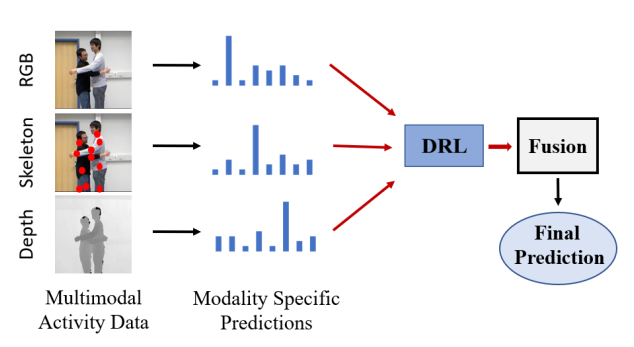

(e)

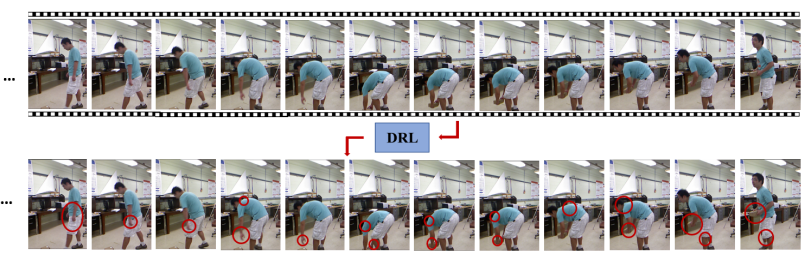

(b)

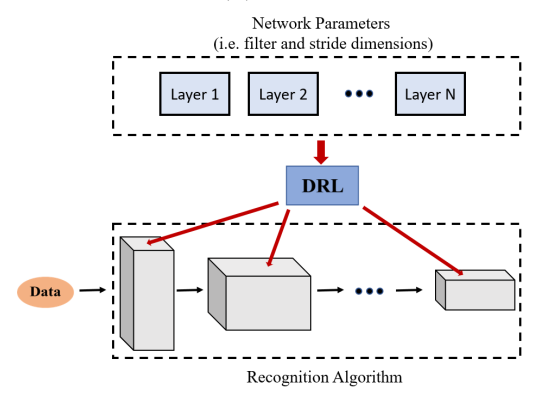

(d)

Figure 1: Examples of using DRL for a) finding temporal attention, b) finding spatial attention, c) early activity prediction, d) finding the best network architecture e) finding fusion weights, f) optimizing the cluster centroids, and g) robot control, to improve the human activity recognition performance.

classification result is correct or not. This has not yet been considered when designing the reward functions.

\section{References}

[Al-Amin et al., 2019] Md Al-Amin, Wenjin Tao, David Doell, Ravon Lingard, Zhaozheng Yin, Ming C Leu, and Ruwen Qin. Action recognition in manufacturing assembly using multimodal sensor fusion. Procedia Manufacturing, 39:158-167, 2019.

[Arulkumaran et al., 2017] Kai Arulkumaran, Marc Peter Deisenroth, Miles Brundage, and Anil Anthony Bharath. Deep reinforcement learning: A brief survey. IEEE Signal Processing Magazine, 34(6):26-38, 2017.

[Dong et al., 2019] Wenkai Dong, Zhaoxiang Zhang, and Tieniu Tan. Attention-aware sampling via deep reinforcement learning for action recognition. In Proceedings of the AAAI Conference on Artificial Intelligence, volume 33, pages 8247-8254, 2019.

[François-Lavet et al., 2018] Vincent François-Lavet, Peter Henderson, Riashat Islam, Marc G Bellemare, and Joelle Pineau. An introduction to deep reinforcement learning. arXiv preprint arXiv:1811.12560, 2018.
[Gao et al., 2019] Yang Gao, Hong Yang, Peng Zhang, Chuan Zhou, and Yue Hu. Graphnas: Graph neural architecture search with reinforcement learning. arXiv preprint arXiv:1904.09981, 2019.

[Gowda et al., 2021] Shreyank N Gowda, Laura SevillaLara, Frank Keller, and Marcus Rohrbach. Claster: clustering with reinforcement learning for zero-shot action recognition. arXiv preprint arXiv:2101.07042, 2021.

[Guo et al., 2021] Jiale Guo, Qiang Liu, and Enqing Chen. A deep reinforcement learning method for multimodal data fusion in action recognition. IEEE Signal Processing Letters, 2021.

[Haque et al., 2016] Albert Haque, Alexandre Alahi, and Li Fei-Fei. Recurrent attention models for depth-based person identification. In Proceedings of the IEEE Conference on Computer Vision and Pattern Recognition, pages 1229-1238, 2016.

[Herath et al., 2017] Samitha Herath, Mehrtash Harandi, and Fatih Porikli. Going deeper into action recognition: A survey. Image and vision computing, 60:4-21, 2017.

[Imran and Raman, 2020] Javed Imran and Balasubramanian Raman. Evaluating fusion of rgb-d and inertial sen- 
sors for multimodal human action recognition. Journal of Ambient Intelligence and Humanized Computing, 11(1):189-208, 2020.

[Jaafra et al., 2019] Yesmina Jaafra, Jean Luc Laurent, Aline Deruyver, and Mohamed Saber Naceur. Reinforcement learning for neural architecture search: A review. Image and Vision Computing, 89:57-66, 2019.

[Jaderberg et al., 2019] Max Jaderberg, Wojciech M Czarnecki, Iain Dunning, Luke Marris, Guy Lever, Antonio Garcia Castaneda, Charles Beattie, Neil C Rabinowitz, Ari S Morcos, Avraham Ruderman, et al. Human-level performance in $3 \mathrm{~d}$ multiplayer games with population-based reinforcement learning. Science, 364(6443):859-865, 2019.

[Ji et al., 2018] Yanli Ji, Yang Yang, Xing Xu, and Heng Tao Shen. One-shot learning based pattern transition map for action early recognition. Signal Processing, 143:364370, 2018.

[Klaser et al., 2008] Alexander Klaser, Marcin Marszałek, and Cordelia Schmid. A spatio-temporal descriptor based on 3d-gradients. In BMVC 2008-19th British Machine Vision Conference, pages 275-1. British Machine Vision Association, 2008.

[Kong and $\mathrm{Fu}, 2018$ ] Yu Kong and Yun Fu. Human action recognition and prediction: A survey. arXiv preprint arXiv:1806.11230, 2018.

[Kumrai et al., 2020] Teerawat Kumrai, Joseph Korpela, Takuya Maekawa, Yen Yu, and Ryota Kanai. Human activity recognition with deep reinforcement learning using the camera of a mobile robot. In 2020 IEEE International Conference on Pervasive Computing and Communications (PerCom), pages 1-10. IEEE, 2020.

[Laptev et al., 2008] Ivan Laptev, Marcin Marszalek, Cordelia Schmid, and Benjamin Rozenfeld. Learning realistic human actions from movies. In 2008 IEEE Conference on Computer Vision and Pattern Recognition, pages 1-8. IEEE, 2008.

[Li, 2017] Yuxi Li. Deep reinforcement learning: An overview. arXiv preprint arXiv:1701.07274, 2017.

[Liu et al., 2019] Zhuo Liu, Chenhui Yao, Hang Yu, and Taihua Wu. Deep reinforcement learning with its application for lung cancer detection in medical internet of things. Future Generation Computer Systems, 97:1-9, 2019.

[Lu et al., 2019] Yantao Lu, Yilan Li, and Senem Velipasalar. Efficient human activity classification from egocentric videos incorporating actor-critic reinforcement learning. In 2019 IEEE International Conference on Image Processing (ICIP), pages 564-568. IEEE, 2019.

[Ma et al., 2021] Yongsen Ma, Sheheryar Arshad, Swetha Muniraju, Eric Torkildson, Enrico Rantala, Klaus Doppler, and Gang Zhou. Location-and personindependent activity recognition with wifi, deep neural networks, and reinforcement learning. ACM Transactions on Internet of Things, 2(1):1-25, 2021.
[Mnih et al., 2013] Volodymyr Mnih, Koray Kavukcuoglu, David Silver, Alex Graves, Ioannis Antonoglou, Daan Wierstra, and Martin Riedmiller. Playing atari with deep reinforcement learning. arXiv preprint arXiv:1312.5602, 2013.

[Mnih et al., 2015] Volodymyr Mnih, Koray Kavukcuoglu, David Silver, Andrei A Rusu, Joel Veness, Marc G Bellemare, Alex Graves, Martin Riedmiller, Andreas K Fidjeland, Georg Ostrovski, et al. Human-level control through deep reinforcement learning. nature, 518(7540):529-533, 2015.

[Nguyen et al., 2017] Ngoc Duy Nguyen, Thanh Nguyen, and Saeid Nahavandi. System design perspective for human-level agents using deep reinforcement learning: A survey. IEEE Access, 5:27091-27102, 2017.

[Nikpour and Armanfard, 2021] Bahareh Nikpour and Narges Armanfard. Joint selection using deep reinforcement learning for skeleton-based activity recognition. In 2021 IEEE International Conference on Systems, Man, and Cybernetics (SMC), pages 1056-1061, 2021.

[Rao et al., 2017] Yongming Rao, Jiwen Lu, and Jie Zhou. Attention-aware deep reinforcement learning for video face recognition. In Proceedings of the IEEE international conference on computer vision, pages 3931-3940, 2017.

[Sallab et al., 2017] Ahmad EL Sallab, Mohammed Abdou, Etienne Perot, and Senthil Yogamani. Deep reinforcement learning framework for autonomous driving. Electronic Imaging, 2017(19):70-76, 2017.

[Sarkar et al., 2022] Arya Sarkar, Avinandan Banerjee, Pawan Kumar Singh, and Ram Sarkar. 3d human action recognition: Through the eyes of researchers. Expert Systems with Applications, page 116424, 2022.

[Shao et al., 2019] Kun Shao, Zhentao Tang, Yuanheng Zhu, Nannan Li, and Dongbin Zhao. A survey of deep reinforcement learning in video games. arXiv preprint arXiv:1912.10944, 2019.

[Sun et al., 2020] Zehua Sun, Qiuhong Ke, Hossein Rahmani, Mohammed Bennamoun, Gang Wang, and Jun Liu. Human action recognition from various data modalities: A review. arXiv preprint arXiv:2012.11866, 2020.

[Sutton and Barto, 2018] Richard S Sutton and Andrew G Barto. Reinforcement learning: An introduction. MIT press, 2018.

[Sutton et al., 2000] Richard S Sutton, Satinder Singh, and David McAllester. Comparing policy-gradient algorithms. IEEE Transactions on Systems, Man, and Cybernetics, 2000.

[Tang et al., 2018a] Yansong Tang, Yi Tian, Jiwen Lu, Peiyang $\mathrm{Li}$, and Jie Zhou. Deep progressive reinforcement learning for skeleton-based action recognition. In Proceedings of the IEEE conference on computer vision and pattern recognition, pages 5323-5332, 2018.

[Tang et al., 2018b] Yansong Tang, Yi Tian, Jiwen Lu, Peiyang Li, and Jie Zhou. Deep progressive reinforcement learning for skeleton-based action recognition. In 
Proceedings of the IEEE Conference on Computer Vision and Pattern Recognition, pages 5323-5332, 2018.

[Torrado et al., 2018] Ruben Rodriguez Torrado, Philip Bontrager, Julian Togelius, Jialin Liu, and Diego PerezLiebana. Deep reinforcement learning for general video game ai. In 2018 IEEE Conference on Computational Intelligence and Games (CIG), pages 1-8. IEEE, 2018.

[Wang et al., 2015] Pichao Wang, Wanqing Li, Zhimin Gao, Jing Zhang, Chang Tang, and Philip O Ogunbona. Action recognition from depth maps using deep convolutional neural networks. IEEE Transactions on Human-Machine Systems, 46(4):498-509, 2015.

[Wang et al., 2016] Aiguo Wang, Guilin Chen, Jing Yang, Shenghui Zhao, and Chih-Yung Chang. A comparative study on human activity recognition using inertial sensors in a smartphone. IEEE Sensors Journal, 16(11):45664578, 2016.

[Wang et al., 2017] Gang Wang, Wenmin Wang, Jingzhuo Wang, and Yaohua Bu. Better deep visual attention with reinforcement learning in action recognition. In 2017 IEEE International Symposium on Circuits and Systems (ISCAS), pages 1-4. IEEE, 2017.

[Wang et al., 2018] Xin Wang, Wenhu Chen, Jiawei Wu, Yuan-Fang Wang, and William Yang Wang. Video captioning via hierarchical reinforcement learning. In Proceedings of the IEEE Conference on Computer Vision and Pattern Recognition, pages 4213-4222, 2018.

[Wang et al., 2019] Weining Wang, Yan Huang, and Liang Wang. Language-driven temporal activity localization: A semantic matching reinforcement learning model. In Proceedings of the IEEE/CVF Conference on Computer Vision and Pattern Recognition, pages 334-343, 2019.

[Weng et al., 2020] Junwu Weng, Xudong Jiang, Wei-Long Zheng, and Junsong Yuan. Early action recognition with category exclusion using policy-based reinforcement learning. IEEE Transactions on Circuits and Systems for Video Technology, 30(12):4626-4638, 2020.

[Wu et al., 2019] Wenhao Wu, Dongliang He, Xiao Tan, Shifeng Chen, and Shilei Wen. Multi-agent reinforcement learning based frame sampling for effective untrimmed video recognition. In Proceedings of the IEEE/CVF International Conference on Computer Vision, pages 62226231, 2019.

[Xiaokai et al., 2019] Chen Xiaokai, Gao Ke, and Cao Juan. Predictability analyzing: Deep reinforcement learning for early action recognition. In 2019 IEEE International Conference on Multimedia and Expo (ICME), pages 958-963. IEEE, 2019.

[Xu et al., 2020] Zheyuan Xu, Yingfu Wang, Jiaqin Jiang, Jian Yao, and Liang Li. Adaptive feature selection with reinforcement learning for skeleton-based action recognition. IEEE Access, 8:213038-213051, 2020.

[Yamagata et al., 2019] Taku Yamagata, Raúl SantosRodríguez, Ryan McConville, and Atis Elsts. Online feature selection for activity recognition using reinforcement learning with multiple feedback. arXiv preprint arXiv:1908.06134, 2019.
[Yeung et al., 2016] Serena Yeung, Olga Russakovsky, Greg Mori, and Li Fei-Fei. End-to-end learning of action detection from frame glimpses in videos. In Proceedings of the IEEE conference on computer vision and pattern recognition, pages 2678-2687, 2016.

[Yun et al., 2017] Sangdoo Yun, Jongwon Choi, Youngjoon Yoo, Kimin Yun, and Jin Young Choi. Action-decision networks for visual tracking with deep reinforcement learning. In Proceedings of the IEEE conference on computer vision and pattern recognition, pages 2711-2720, 2017.

[Zoph and Le, 2016] Barret Zoph and Quoc V Le. Neural architecture search with reinforcement learning. arXiv preprint arXiv:1611.01578, 2016. 